\title{
A modified method for the treatment of urethral fistula after hypospadias repair
}

\author{
Chao Qin*, Wei Wang*, Shang-Qian Wang, Qiang Cao, Zeng-Jun Wang, Peng-Chao Li, Jie Li, Ning-Han Feng, \\ Li-Xin Hua, Chang-Jun Yin and Wei Zhang
}

Urethrocutaneous fistula (UCF) is a common complication of hypospadias surgery for severe hypospadias. We report our experience in the management of UCF following hypospadias surgery with a prepuce-degloving method (PDM). Our study included 87 patients who developed UCF after hypospadias repair from May 2001 to December 2011. Either simple closure or PDM was performed to repair the fistula. In total, 61 patients underwent a simple closure or $Y-V$ plasty of the fistula, and 26 underwent a PDM repair. The success rate was $78.7 \%$ for simple closure or Y-V plasty and $\mathbf{9 6 . 2 \%}$ for PDM repair $(P<0.05)$. PDM repair represents a good choice for UCF repair after hypospadias, and our high $96.2 \%$ success rate demonstrates its applicability.

Asian Journal of Andrology (2012) 14, 900-902; doi:10.1038/aja.2012.85; published online 15 October 2012

Keywords: hypospadias; repair; urethrocutaneous fistula

\section{INTRODUCTION}

Hypospadias surgery is one of the most difficult areas in pediatric urology and it has been characterized by constant evolution. Some of the surgical techniques that have been proposed in the past are now considered inadequate because of an unacceptable complication rate or poor functional and aesthetic results. A total of $55 \%$ of the proximal hypospadiac patients and $43 \%$ of those with multiple operations were very dissatisfied with the appearance and size of their penis. ${ }^{1}$ The postoperative appearance of the urethrocutaneous fistula (UCF) remains one of the most frustrating complications after hypospadias surgery. In an early report, 253 consecutive hypospadias were repaired using the Byars technique, and $22 \%$ had postoperative fistula. ${ }^{2}$ The use of buccal mucosa grafts in complex hypospadias patients could result in UCF in up to $25 \%{ }^{3}$ However, experience to date has led to a general consensus with regard to the indications, timing and surgical principles for repair of the hypospadiac meatus. Adaptations to the technique, including strict adherence to the principles of plastic surgery in tissue handling, avoiding suture line overlap and the transposition of additional tissue layers, have cumulatively contributed to minimizing the incidence of failure. During the last decade, different repair methods have been performed according to the location and size of the fistula. A minimal UCF can be repaired using a simple suture or Y-V plasty, ${ }^{4}$ and severe malformation can be treated by techniques including Mathieu repair and so on, as described in the literature. ${ }^{5-9}$ However, the incisions selected in these techniques are all near the fistula, with reported failure rates varying from $5 \%$ to $47 \%{ }^{6}$ The aim of this study was to evaluate a new method for the management of UCF by performing a prepuce-degloving method (PDM), with an incision at the penile coronary sulcus without considering the location and size of the fistula.

\section{PATIENTS AND METHODS}

\section{Patients}

Our study included 87 patients, who developed UCF after hypospadias repair from May 2001 to December 2011. In total, 61 patients had a simple closure or Y-V plasty of the fistula (group A) using little dartos from the surrounding tissues, whereas 26 had a PDM repair (group B). Among these 87 patients, 24 cases had severe fistula that were more than $1 \mathrm{~cm}$ in calibre and 63 cases were less than $1 \mathrm{~cm}$. Moreover, there were 71 patients with one orificium fistula and the rest had two orificium fistula, all located on the ventral aspect of the penis. The reconstruction of the UCF should be carried out at least 3 months after the hypospadias surgery. Of the patients in this study, 52 received their first fistula repair, 24 cases were treated for the second time and 10 were treated for the third time. Only one patient underwent repair for the sixth time. The mean age and the distribution of the clinical characteristics between the two groups are presented in Table $\mathbf{1 .}$

\section{Surgical technique}

All the procedures were performed in accordance with the ethical standards of the committee on human experimentation of the Nanjing Medical University. The fistula opening location was confirmed and a ureteral catheter was inserted as a mark before the surgery (Figure 1a). Then, the catheter was inserted from the urinary meatus. A tourniquet was used at the root of the penis (Figure 1b). The circumcision of the retracted prepuce inner plate was $0.5 \mathrm{~cm}$ near the coronary sulcus and close to Buck's fascia of the penis. Isolation of a flap with a complete blood supply was performed toward the proximate penis along the albuginea penis and across the fistula pedicle (about approximately $1-2 \mathrm{~cm}$ beyond the exterior orificium fistula) 
Table 1 Distribution of clinical characteristics between simple suture group and PDM group

\begin{tabular}{lccc}
\hline Variables & Group A & Group B & P* \\
\hline Patients, $n(\%)$ & $61(70 \%)$ & $26(30 \%)$ & \\
Age (year) & $7.2 \pm 3.1$ & $8.0 \pm 1.9$ & 0.061 \\
Meatal location & & & \\
$\quad$ Distal & 21 & 10 & 0.442 \\
$\quad$ Midpenile & 23 & 12 & \\
$\quad$ Proximal & 20 & 17 & \\
Meatal size & & & 0.665 \\
$\quad<1 \mathrm{~cm}$ & 45 & 18 & \\
$\quad 1 \mathrm{~cm}$ & 16 & 8 & \\
Meatal numbers & & & \\
1 & 51 & 20 & \\
2 & 10 & 6 & \\
Previous repair & & & \\
$\quad$ No & 36 & 16 & \\
$\quad$ Yes & 25 & 10 & \\
\hline
\end{tabular}

Abbreviation: PDM, prepuce-degloving method.

*t-test for age distribution between group $\mathrm{A}$ and group $\mathrm{B}$; two-sided $\chi^{2}$ test for other selected variables between the two groups.

(Figure 1c) without damaging the new artificial urethra. (Figure 1d). Subsequently, the fistula was cut close to the urethra and 5-0 Vicryl thread was used to suture the orificium fistula (Figure 1e). Thereafter, near the fistula, a semicircular Buck's fascia pedicle flap that was taken from the surface of the albuginea penis and was larger than the fistulous opening was turned over to cover the sutured wound surface of the fistula (Figure 1f). At the same time, severe external fistula opening was closed by suture of the subcutaneous tissue from the internal sheath of the prepuce using 5-0 line (Figure 1g). The dissociative sheath was anastomosed with the prepuce of the coronary sulcus so that the prepuce of the external fistula could be drawn close to the internal fistula opening. Finally, the foreskin was dressed with pressure and the tourniquet was removed (Figure 1h). After 1 week, the catheter was removed to permit recovery of urination.

\section{RESULTS}

The clinical and demographic factors of the two groups are presented in Table 1. No significant differences in the distribution of age, fistula location and size between the two groups have been observed. A simple closure or Y-V plasty of the fistula was performed on 61 patients (group A). A total of 26 boys (mean age 8 years old) in group B underwent PDM repair of UCF at our hospital during the study period. After the first repair, the fistula recurred in 13 boys in group A and one in group B. The overall success rates of first repair were $78.7 \%$ and $96.2 \%$, respectively $(P<0.05)$. In the second repair, the same technique was used as was performed for the first repair. After the second repair, there was no recurrence in group $B$, while there was recurrence in six of 13 boys in group A. PDM repair was used in these boys and no recurrence was observed. To date, after a mean follow-up of 4.5 years (range 0.3-9.5 years), there have been no further recurrences.

\section{DISCUSSION}

The successful repair of UCF depends on several basic principles, including the avoidance of performing the procedure on inflamed or edematous tissue (hence an interval of $\geqslant 6$ months between any interventions), the correction of any urethral or meatal obstruction distally, a tension-free urethral closure with an absorbable suture material and coverage of the urethral repair with well-vascularised tissue. During the past decades, we have explored reconstruction
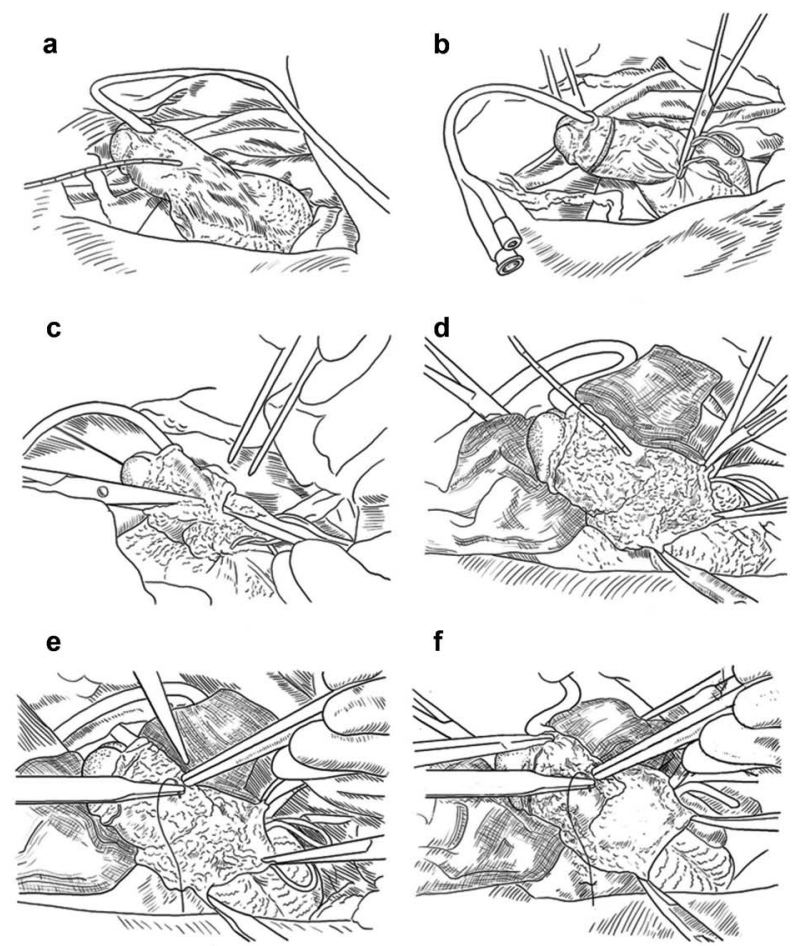

f
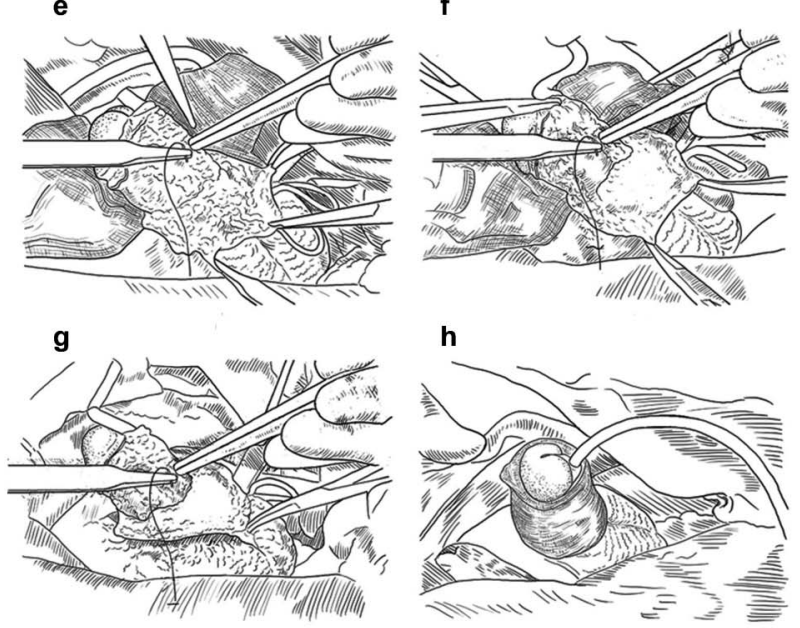

Figure 1 Operating procedure of the sleev-resected-prepuce technique. (a) The fistula location was confirmed before the surgery; (b) Tourniquet was used at the root of the penis; (c) Ring-shaped incision of the prepuce inner plate and isolation of foreskin was performed toward the proximate glans; (d) Pedicle of the fistula was isolated; (e) The fistula was cut and the orificium fistula was sutured; (f) Fascio-cutaneous flap was turned over to cover the wound surface of fistula; (g) Subcutaneous tissue was sutured from the internal sheath of prepuce to close the orificium fistula; (h) External genital appearance at the completion of skin closure.

methods to improve the rate of failure of repair surgery. For minimal UCF, a simple suture or local Y-V plasty was carried out according to previous experience. ${ }^{4}$ However, the higher incidence of a local blood supply shortage may lead to the formation of a UCF or even complete failure of the surgery. For severe UCF, Mathieu repair was performed for orificium fistula located at the distal aspect of the penis when sufficient tissues were present under the fistula. ${ }^{5}$ For cases with less skin on the ventral side of the penis, such that the tube could not be sutured, Snodgrass repair has typically been carried out, although the success rate reported by Djordjevic ${ }^{10}$ was only $83 \%$. The Duckett approach ${ }^{8}$ has been applied when prepuce abundance in the dorsal region of the penis is accompanied by obvious chordee of the penis, whereas Onlay island flap method can be used in cases in which the integrity of the urethral plate is retained and without penile recurvation. ${ }^{9}$

Despite great care taken during the procedure, UCF may recur after repair, and the recurrence rates that have been reported vary from $15 \%$ to $47 \% .{ }^{6,11}$ Except for those with large ( $>2 \mathrm{~mm}$ ) or multiple fistula, we 
found that no specific feature rendered recurrence more or less likely, and the chance of recurrence was not substantially influenced by the means of skin closure. It is uncertain why some fistula recurs in a minority, and multiplies. However, some factors, such as blood supply, infection, tension and distal obstruction, might increase the risk of recurrence. In our opinion, beyond any deficiencies of the surgical technique or postoperative management, there is no clear explanation for recurrence other than the impaired local vascularity which might be the most plausible explanation. Furthermore, the accumulation of secretions, stricture or obstruction of the urethra, repeated penile erection and wound infection might influence the failure risk, but further studies are needed. As the incision site was near the fistula, failure of the repair may cause single deformity to become severe or cause one fistula to develop into more.

Based on the above-mentioned studies, we have completely replaced the traditional surgical method with a PDM repair in which the incisions are not made near the orificium fistula, reducing the risk of interference with the blood supply, complications related to tension and the occurrence of infection. There are three main advantages of this technique over the traditional methods. First, traditional surgical concepts involving simple repair and local flap grafting can be replaced by complete isolation and resection of the fistulous tract after sleeve of the prepuce. Second, the dissociative prepuce sheath is anastomosed with the coronary sulcus. The outer layer of the prepuce suture lines does not overlap the fistula sutures so that normal prepuce tissue can completely cover the orificium fistula, avoiding the placement of an incision near the fistula, reducing the incidence of blood supply reduction, local tension and infection. Finally, a fascio-cutaneous flap with a pedicle dartos flap is turned over to cover the sutured wound surface of the fistula, which may also improve the outcome of the surgery.

Hypospadias is the most common congenital anomaly of the penis. ${ }^{12}$ The PDM repair represents a good choice for UCF after hypospadias surgery, and our high success rate of $96.2 \%$ demonstrates its applicability. Therefore, the great value of this technique in reducing the notably high incidence in failure of UCF repair warrants wide recommendation of this technique.

\section{AUTHOR CONTRIBUTIONS}

All authors discussed the results and implications and commented on the manuscript at all stages. ZJW, LXH, CJY and WZ conceived and designed the study. CQ, QC and SQW gathered and analyzed the data. CQ, PCL, JL, NHF and WW performed the operations. CQ, WW and SQW wrote and revised the paper, which was read and approved by all authors.

\section{COMPETING FINANCIAL INTERESTS}

The authors declare no competing financial interests.

\section{ACKNOWLEDGMENTS}

This study was supported by the National Nature Science Foundation of China (No. 30872575), the Health Project of Jiangsu Province (No. XK1720090) and the National Eleventh-Five Science and Technology Support Program of China (No. 2006BAI03B12).

1 Wang WW, Tu XA, Deng $\mathrm{CH}$, Mo JC, Zhao $\mathrm{L}$ et al. Long-term sexual activity status and influencing factors in men after surgery for hypospadias. Asian J Androl2009; 11:417-22.

2 Wray RC Jr, Ribaudo JM, Weeks PM. The Byars hypospadias repair. A review of 253 consecutive patients. Plast Reconstr Surg 1976; 58: 329-31.

3 Irani D, Hekmati P, Amin-Sharifi A. Results of buccal mucosal graft urethroplasty in complex hypospadias. Urol J 2005; 2: 111-4.

4 Goldstein HR, Hensle TW. Simplified closure of hypospadias fistulas. Urology 1981; 18: 504-5.

5 Kureel SN, Vasudeva P, Sinha SK, Dalela D. "Limited" double dorsal dartos flap coverage. An effective alternative to conventional flap coverage of the neourethra following Mathieu repair for subcoronal hypospadias. Int Urol Nephrol 2008; 40: 569-72.

6 Eardley I, Whitaker RH. Surgery for hypospadias fistula. Br J Urol 1992; 69: 306-10.

7 Ye WJ, Ping P, Liu YD, Li Z, Huang YR. Single stage dorsal inlay buccal mucosal graft with tabularized incised urethral plate technique for hypospadias reoperations. Asian $J$ Androl 2008; 10: 682-6.

8 Karamursel S, Celebioglu S. Urethral advancement for recurrent distal hypospadias fistula treatment. Ann Plast Surg 2006; 56: 423-6.

9 Shankar KR, Losty PD, Hopper M, Wong L, Rickwood AMK. Outcome of hypospadias fistula repair. BJU Int 2002; 89: 103-5.

10 Djordjevic ML, Perovic SV, Slavkovic Z, Djakovic N. Longitudinal dorsal dartos flap for prevention of fistula after a Snodgrass hypospadias procedure. Eur Urol 2006; 50: 53-7.

11 Hayashi Y, Kojima Y, Kurokawa S, Mizuno K, Nakane A et al. Scrotal dartos flap for the prevention of the urethrocutaneous fistula on hypospadias urethroplasty. Int J Urol 2005; 12 : 280-3.

12 Leung AK, Robson WL. Hypospadias: an update. Asian J Androl 2007; 9: 16-22. 